\title{
Heating of Hollow Photonic Bragg Fibers From Field Propagation, Coupling, and Bending
}

\author{
M. Skorobogatiy, S. A. Jacobs, S. G. Johnson, C. Anastassiou, and B. Temelkuran
}

\begin{abstract}
We investigate heating from field propagation, coupling, and bending, which are the potential failure mechanisms for an emerging new type of high-power radiation guides-hollow photonic Bragg fibers. Continuous wave $(\mathrm{CW})$ and pulsed radiation sources are considered, assuming continuous operation of the laser source.
\end{abstract}

Index Terms-Bragg fiber, high-laser-power transmission, photonic bandgap fiber, power capacity.

\section{INTRODUCTION}

$\mathbf{L}$ ARGE-CORE hollow dielectric fibers have been recently investigated to provide a guiding platform for high-power radiation in a wide range of $I R$ frequencies from 1 to $10.6 \mu \mathrm{m}$ [1]-[5]. Due to the absence of an absorbing material in the core, hollow fibers have a potential to guide kilowatts of continuouswave $(\mathrm{CW})$ power at a designable $I R$ wavelength.

To transfer this novel technology into the industrial domain, one has to deal with a variety of factors limiting the power capacity of such fibers. In high-power-guiding applications (Fig. 1), major areas of theoretical and experimental development are laser-fiber coupling efficiency; reduction of heating at the input fiber face; straight fiber design for minimized radiation and material-absorption losses; reduction of mode scattering and radiation due to imperfections and bends; suppression of excess heating due to bends and imperfections; and control of the output beam quality, which degrades due to bends and imperfections. In this paper, we will focus on issues related to the heating of hollow Bragg fibers (HBFs) operating with the linearly polarized $\mathrm{HE}_{11}$ mode, which is the most suitable mode to be coupled into with a standard highpower laser.

HBF consists of a hollow core, a series of bilayers of two materials with sufficient index contrast, and an overcladding. Fig. 2(a) is a schematic of an HBF cross section. Hollow-core radius is $R_{i}$. The dielectric mirror has $N$ bilayers extending to a radius $R_{m}$. Each bilayer is of thickness $d$. The outer radius of the overcladding is $R_{o}$. Typically, $R_{i} \sim R_{m} \ll R_{o}$. The electromagnetic field decays exponentially in the mirror.

Manuscript received November 1, 2004; revised June 22, 2005. This work was supported by the Department of Defense (DOD)-Small Business Innovation Research (SBIR) under Air Force (AF) Grant F49620 02-D-0089.

M. Skorobogatiy is with Ecole Polytechnique de Montréal, Centre-ville, Montréal, QC H3C 3A7, Canada (e-mail: maksim.skorobogatiy @ polymtl.edu).

S. A. Jacobs, C. Anastassiou, and B. Temelkuran are with OmniGuide Communication, Cambridge, MA 02139 USA

S. G. Johnson is with the Department of Applied Mathematics, Massachusets Institute of Technology, Cambridge, MA 02139 USA.

Digital Object Identifier 10.1109/JLT.2005.857778

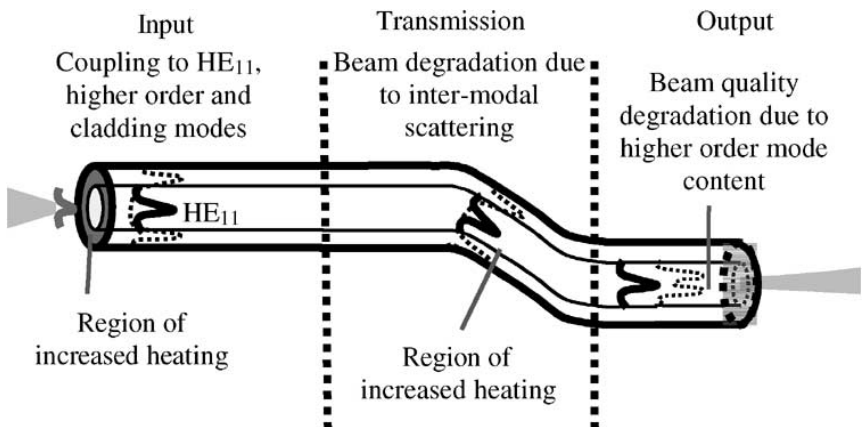

Fig. 1. Major development areas in a high-power fiber link. Input-coupling efficiency, input face heating. Transmission-design and optimization of a straight fiber, mitigation of scattering/radiation due to imperfections/bends, excess heating due to bends. Output—control of beam quality and $M^{2}$.

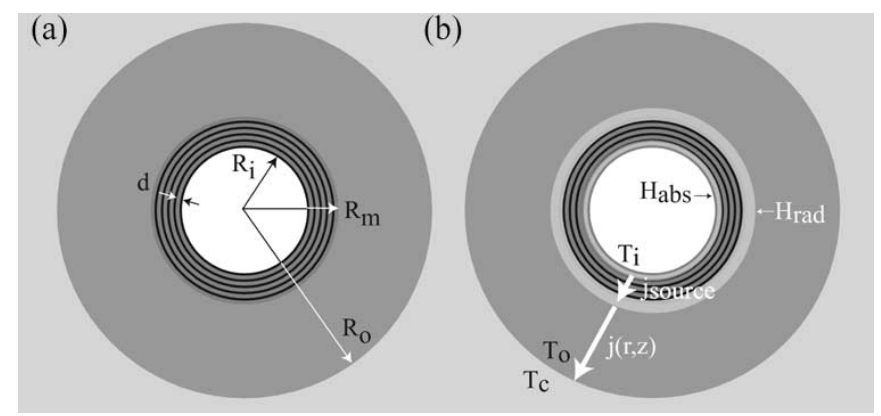

Fig. 2. Power-capacity analysis of an HBF. (a) Schematic of a fiber. Hollow core of radius $R_{i}$, mirror region of $N$ bilayers, each of thickness $d$, surrounded by an overcladding extending to $R_{o}$. (b) Two major types of heat sources (shown in light gray) $-H_{\mathrm{abs}}$ is due to field penetration and absorption in the mirror region, $H_{\text {rad }}$ is due to radiation leakage through a finite-size mirror and absorption in the overcladding.

Thus, field penetration into the mirror is typically limited to the first few bilayers. Since the core area that guides most of the light is several orders of magnitude larger than the mirror area, power dissipation due to material absorption $H_{\mathrm{abs}}$ is greatly suppressed, with most of the heating happening in the first few mirror layers [shown in light gray in Fig. 2(b)]. Another mechanism of power dissipation in HBFs is radiation $H_{\mathrm{rad}}$ leaking through the finite-size mirror. If the overcladding region is absorbing, most of the radiation escaping from the core will be converted into heat in that region. Sometimes, when absorption in the overcladding is very high $\left(10^{5} \mathrm{~dB} / \mathrm{m}\right.$ for a polymer at $10.6 \mu \mathrm{m}$ ), all the heat conversion occurs in a thin circular region at $R_{\mathrm{m}}$ just outside the mirror [also shown in light gray on Fig. 2(b)]. Assuming no hear transfer to the hollow 
core, all the heat dissipation occurs through the overcladding periphery at $R_{o}$. Cooling there is provided by conduction and convection to gas or flowing water at an operating temperature $T_{c}$. Heat transfer from fiber to coolant is characterized by a heat transfer coefficient $h$, which can depend strongly on the fiber diameter. Typical heat-transfer coefficients for a solid-gas/liquid flat interface are dry air- $h=5 \mathrm{~W} /\left(\mathrm{m}^{2} \cdot \mathrm{K}\right)$, forced dry air$h=25 \mathrm{~W} /\left(\mathrm{m}^{2} \cdot \mathrm{K}\right)$, water- $h=200 \mathrm{~W} /\left(\mathrm{m}^{2} \cdot \mathrm{K}\right)$, while the same coefficients for fibers with outer diameters of $\sim 1 \mathrm{~mm}$ can be five to ten times larger. Common heat conductance of polymers and glasses is $k \sim 0.25 \mathrm{~W} /(\mathrm{m} \cdot \mathrm{K})$.

A typical design for $\lambda=10.6 \mu \mathrm{m}$ has $R_{i}=500 \mu \mathrm{m}, R_{o}=$ $1500 \mu \mathrm{m}$, and 14 bilayers. The mirror materials are polymer (low index) and chalcogenide glass (high index), with indices of $n_{l}=1.5$ and $n_{h}=2.8$, respectively. Bilayer thickness is $d=$ $3 \mu \mathrm{m}$. Intrinsic bulk absorption loss of a high-index material is $\sim 10 \mathrm{~dB} / \mathrm{m}$, an overestimate for high-purity chalcogenide glasses at $10.6 \mu \mathrm{m}$. The overcladding and low-index materials are assumed to have absorption losses of $10^{5} \mathrm{~dB} / \mathrm{m}$, which is a value typical for polymers. These material parameters yield a total $\mathrm{HE}_{11}$ modal loss of $\alpha_{\mathrm{HE}_{11}} \sim 0.1 \mathrm{~dB} / \mathrm{m}$. For this design, total power dissipation is dominated by material-absorption losses in the first few layers of the mirror.

\section{Heating With a CW Laser Source}

We first analyze the heating of a fiber transmitting $P[\mathrm{~W}]$ of power coming from a $\mathrm{CW}$ laser source. For $\mathrm{CW}$ sources, temperature rise in a fiber is very sensitive to the cooling conditions, with most of the temperature rise occurring at the cladding to air/water interface, and only a small temperature difference across the fiber profile. A semirigorous analysis of temperature increase in hollow fibers and preforms for $\mathrm{CW}$ sources, typically ignores intermodal interferences, and can be encountered in the literature on hollow metallic fibers [6]-[9]. In this paper, we present rigorous formulation for both $\mathrm{CW}$ and pulsed sources.

\section{A. Simple Model}

The equilibrium temperature profile can be found by solving the standard heat-transfer equation, with the sources determined by the modal profile in the absorbing regions of a fiber. For a single propagating mode, we find that the solution of a heattransfer equation with exact sources can be approximated very well by a simpler model. Instead of an exact heat source, we assume a thin circular heat source $H_{\text {abs }}+H_{\text {rad }}$ at $R_{i}$ with a heat flux through the core $\left.j_{r}(r, z)\right|_{r=R_{i}}=P_{m} \alpha_{m} \exp \left(-\alpha_{m} z\right)$, where $P_{m}$ is the partial power and $\alpha_{m}$ is the total loss of a mode $m$. Boundary conditions assume zero heat flux through the fiber facets $\left.j_{r}(r, z)\right|_{z=0}=\left.j_{r}(r, z)\right|_{z=L}=0$. Finally, we use the Newton boundary condition for the outgoing heat flux into the coolant in the form $\left.j_{r}(r, z)\right|_{r=R_{o}}=h\left(T\left(R_{o}, z\right)-T_{c}\right)$. In a simple analysis, we ignore the interference effects between copropagating modes, thus adding their individual contributions to the temperature rise. In this model, the most heated region of a fiber is at the fiber-coupling facet, near the inner mirror edge, with a corresponding temperature rise $\Delta T^{\mathrm{CW}}=T_{i}-T_{c}$

$$
\begin{array}{r}
\Delta T^{\mathrm{CW}}=\left(\sum_{R_{i} \alpha_{m} \leqslant 1} P_{m} \alpha_{m}\right)\left(\frac{1}{2 \pi R_{o} h}+\frac{\log \left(\frac{R_{o}}{R_{i}}\right)}{2 \pi k}\right) \\
+\left(\sum_{R_{i} \alpha_{m}>1} P_{m} \frac{2 \log \left(R_{i} \alpha_{m}\right)}{\pi^{2} R_{i} k}\right) .
\end{array}
$$

The first summation in (1) accounts for the core-guided modes, each of which has relatively small modal losses and modal decay occurring on a length scale larger than the core radius $R_{i} \alpha_{m} \leqslant 1$. These modes are relatively benign, as heating from the dissipation of such modes can be counteracted by improved cooling. When coupling to these fiber modes, one has to avoid coupling to the very lossy overcladding modes $R_{i} \alpha_{m}>1$, for which all the power is immediately converted into the heat in a small spatially localized region near the input fiber facet. Heating from such modes cannot be effectively counteracted, as the heated region is smaller than the fiber cross section, rendering heat dissipation through the outer boundary ineffective. We find that the optimal coupling of power from the Gaussian laser beam to the $\mathrm{HE}_{11}$ mode of a fiber is $P_{\mathrm{HE}_{11}} \approx$ 0.98 , and it is achieved when the diameter of the laser beam is $\sim 0.6$ times the fiber diameter, with coupling to the very lossy overcladding modes being $P_{\text {cladding }} \approx 10^{-3}$. Choosing a laser-beam diameter smaller than the optimal, while reducing coupling to the $\mathrm{HE}_{11}$ mode, also greatly reduces the coupling to the overcladding modes, with the rest of the power going to the relatively low-loss higher order core-guided modes. Thus, with a laser-beam diameter of 0.3 times the core diameter, power in the $\mathrm{HE}_{11}$ mode is still large $\left(P_{\mathrm{HE}_{11}} \approx 0.65\right)$, while power in the overcladding modes is less than $P_{\text {cladding }} \approx 10^{-6}$. Thus, using smaller-waist laser beams at the coupling facet of the fiber can substantially reduce fiber heating at the expense of the deterioration of the beam quality. These theoretical results on coupler efficiency and heating were also confirmed by direct experimental observations.

\section{B. Rigorous Formulation}

Although simple model (1) is adequate to quantify fiber heating on average, it is inadequate to describe an observable localized heating rise along the direction of propagation at the points of constructive interference of copropagating modes. To describe this phenomenon, we have to resort to a complete formulation of the heat-transfer problem. Particularly, we assume laser-beam excitation of several fiber modes counted by index $i$ and angular-momentum index $m$, with excitation coefficients $C_{i, m}(z)$ along the length of a fiber. At $z=0$, these coefficients are found by solving a laser beam-fiber coupling problem with the proper field-continuity conditions on the fibercoupling facet. Particularly, the incident laser beam, assumed to have a Gaussian profile, is decomposed into a sum of forwardpropagating modes of a homogeneous waveguide, and the reflected field is expressed as a sum of backward-propagating 


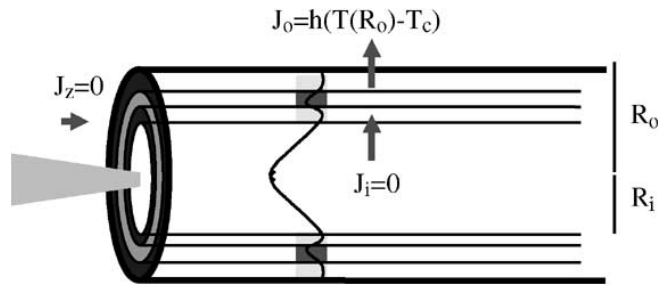

Fig. 3. Modeling of fiber heating. Most heating comes from energy dissipation in the mirror layers made of highly absorbing material. This dissipation defines a very nonuniform heat source in the radial direction. Heat transfer to the core and through the fiber end facets is assumed zero, with all the heat dissipating through the outer surface of a fiber.

free space modes. The amplitudes of the modes are chosen to satisfy transverse field continuity.

Due to the modal interference, the most heated region in the fiber is not necessarily at the coupling facet of the fiber, but is determined by a particular modal-excitation pattern and is usually somewhere near the coupling facet. Equilibrium temperature in this region can be found by solving a timeindependent heat-transfer equation with a boundary condition of zero flux across the fiber end facets and into the fiber core. The heat source is computed exactly from the excited modal pattern. Energy loss in the layers made of higher absorptionloss material will contribute most to the heating; therefore, the heat source will be a rapidly varying function in the radial direction (Fig. 3).

Modal fields of a Bragg fiber have a general form

$$
\left(\begin{array}{c}
\mathbf{E}(\rho, \theta, z, t) \\
\mathbf{H}(\rho, \theta, z, t)
\end{array}\right)=\exp \left(i\left(\beta_{i} z-\omega t\right)\right) \exp (i m \theta)\left(\begin{array}{c}
\mathbf{E}^{i, m}(\rho) \\
\mathbf{H}^{i, m}(\rho)
\end{array}\right) \text {. }
$$

In the following analysis, we assume linearly polarized modes. Particularly, one can show that any mode with $m>0$ has a degenerate pair with the same propagation constant but a negative angular momentum $-m$. Any linear combination of such modes will also be an eigenstate degenerate with the constituent modes. One typically defines the following linear combinations of the modes (we call them "+" and "-" like polarized modes) of index $i$ and angular momenta $m$ and $-m$, which are most compatible with a polarization of an incoming linearly polarized laser mode shown in (3), shown at the bottom of the page. The total field in the fiber is then

$$
\mathbf{F}(\rho, \theta, z)=\sum_{i, m} C_{i, m}^{ \pm}(z) \exp \left(i \beta_{i, m} z\right) \mathbf{F}_{ \pm}^{i, m}(\rho, \theta)
$$

where $\mathbf{F}$ is either an electric- or magnetic-field vector. In the case of straight-fiber, expansion coefficients, $C_{i, m}^{ \pm}(z)=C_{i, m}^{ \pm}$ are independent of $z$ and reflect initial excitation weights. In general, in the case of imperfect fibers (for example, bent fibers), modal expansion coefficients are changing along the propagation length and have to be computed beforehand using coupled-mode theory. The total energy flux along the fiber direction is normalized by the total incoming power $P_{z}(0)=$ $P[\mathrm{~W}]$ and is equal to

$$
\begin{aligned}
& P_{z}^{\mathrm{CW}}(z) \\
& =\frac{1}{4} \hat{Z} \cdot \oint_{\substack{\text { waveguide } \\
\text { cross section }}}\left(\mathbf{H}^{*} \times \mathbf{E}+\mathbf{H} \times \mathbf{E}^{*}\right) \mathrm{d} s \\
& =\frac{1}{4} \sum_{i, j, m}\left(\eta_{m} C_{i, m}^{* \pm}(z) C_{j, m}^{ \pm}(z) P_{z}^{i, j, m}\right. \\
& \left.\quad \cdot \exp \left(\left[-\operatorname{Im}\left(\beta_{j, m}+\beta_{i, m}\right)+i \operatorname{Re}\left(\beta_{j, m}-\beta_{i, m}\right)\right] z\right)\right)
\end{aligned}
$$

where $\eta_{0}=1, \eta_{m \neq 0}=1 / 2$, and a cross flux is defined as

$$
\begin{aligned}
P_{z}^{i, j, m}=\hat{Z} \cdot \int_{0}^{+\infty} 2 \pi \rho \mathrm{d} \rho\left(\mathbf{H}_{i, m}^{*}(\rho) \times \mathbf{E}_{j, m}(\rho)\right. & \\
& \left.+\mathbf{H}_{j, m}(\rho) \times \mathbf{E}_{i, m}^{*}(\rho)\right) .
\end{aligned}
$$

The heat flux from the material absorption of the electric field is calculated by using an imaginary part of the material dielectric constant as

$$
\begin{aligned}
& S^{\mathrm{CW}}(\rho, \theta, z) \\
&= \frac{\operatorname{Im}(\varepsilon(\rho)) \omega}{2 c}|\mathbf{E}|^{2} \\
&= \frac{\operatorname{Im}(\varepsilon(\rho)) \omega}{4 c} \\
& \cdot \sum_{m, m^{\prime}} \sum_{i, j}\left(C_{i, m}^{* \pm}(z) C_{j, m^{\prime}}^{ \pm}(z)\right. \\
& \cdot \exp \left(\left[-\operatorname{Im}\left(\beta_{j, m^{\prime}}+\beta_{i, m}\right)\right.\right. \\
&\left.\left.\quad+i \operatorname{Re}\left(\beta_{j, m^{\prime}}-\beta_{i, m}\right)\right] z\right) \\
& \cdot\left(\cos \left(\left(m-m^{\prime}\right) \theta\right) \chi^{i, j, m-m^{\prime}}\right. \\
&\left.\left. \pm \cos \left(\left(m+m^{\prime}\right) \theta\right) \chi^{i, j, m+m^{\prime}}\right)\right)
\end{aligned}
$$

where one chooses the + sign for " + " polarized modes and the - sign for "-" polarized ones, and

$$
\begin{aligned}
\chi^{i, j, m-m^{\prime}} & =\left(E_{z}^{* i, m} E_{z}^{j, m^{\prime}}+E_{\rho}^{* i, m} E_{\rho}^{j, m^{\prime}}+E_{\theta}^{* i, m} E_{\theta}^{j, m^{\prime}}\right) \\
\chi^{i, j, m+m^{\prime}} & =\left(E_{z}^{* i, m} E_{z}^{j, m^{\prime}}+E_{\rho}^{* i, m} E_{\rho}^{j, m^{\prime}}-E_{\theta}^{* i, m} E_{\theta}^{j, m^{\prime}}\right) .
\end{aligned}
$$

$$
\begin{aligned}
& \left(\begin{array}{l}
\mathbf{E}_{+}^{i, m}(\rho, \theta) \\
\mathbf{H}_{+}^{i, m}(\rho, \theta)
\end{array}\right)=\left(\begin{array}{c}
-\cos (\theta m) E_{z}^{i, m}(\rho),-\cos (\theta m) E_{\rho}^{i, m}(\rho), \sin (\theta m) E_{\theta}^{i, m}(\rho) \\
\sin (\theta m) H_{z}^{i, m}(\rho), \sin (\theta m) H_{\rho}^{i, m}(\rho),-\cos (\theta m) H_{\theta}^{i, m}(\rho)
\end{array}\right) \\
& \left(\begin{array}{l}
\mathbf{E}_{-}^{i, m}(\rho, \theta) \\
\mathbf{H}_{-}^{i, m}(\rho, \theta)
\end{array}\right)=\left(\begin{array}{c}
\sin (\theta m) E_{z}^{i, m}(\rho), \sin (\theta m) E_{\rho}^{i, m}(\rho), \cos (\theta m) E_{\theta}^{i, m}(\rho) \\
\cos (\theta m) H_{z}^{i, m}(\rho), \cos (\theta m) H_{\rho}^{i, m}(\rho), \sin (\theta m) H_{\theta}^{i, m}(\rho)
\end{array}\right)
\end{aligned}
$$




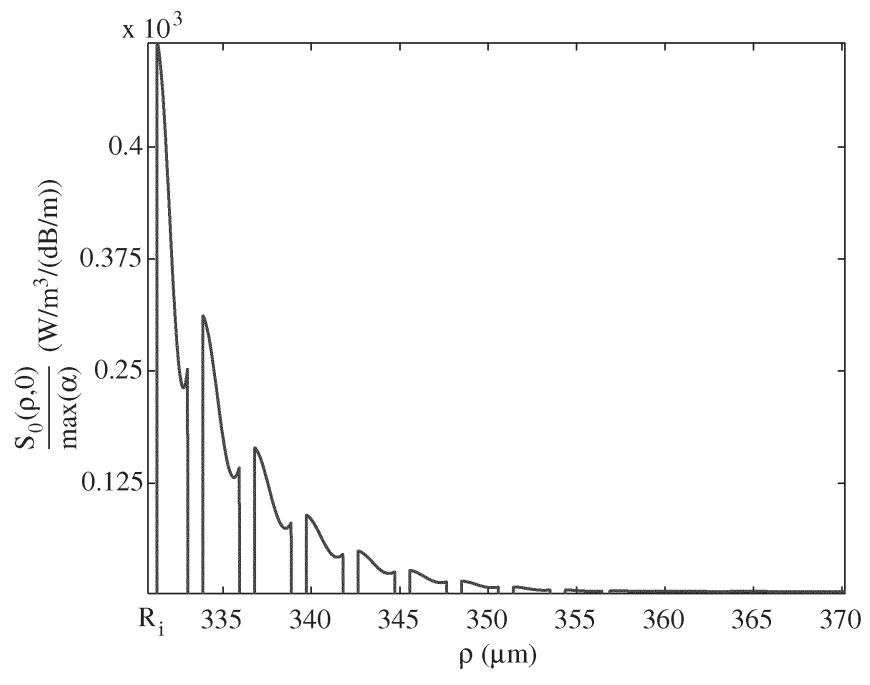

Fig. 4. Heat source $S_{m}^{\mathrm{CW}}(\rho, 0)$ of $\mathrm{HE}_{11}$ mode in a fiber mirror region. The radius of a fiber core is $R_{i}=330 \mu \mathrm{m}$. Most of the heating comes from energy dissipation in the mirror layers made of a polymer material. Due to the exponential decay of a modal field inside a reflector, most of the heat is dissipated in the first few mirror layers.

We define $T(\rho, \theta, z)$ to be the temperature distribution across the fiber profile. Angular dependence of the heat source suggests that temperature distribution can be represented in the following form $T(\rho, \theta, z)=T_{c}+\sum_{m} T_{m}(\rho, z) \cos (m \theta)$, where $T_{\mathrm{c}}$ is a coolant temperature. For every angular component of a temperature distribution, we then write a corresponding heattransfer equation in cylindrical coordinates

$$
\begin{array}{r}
\left(\frac{\partial^{2}}{\partial \rho^{2}}+\frac{1}{\rho} \frac{\partial}{\partial \rho}-\frac{m^{2}}{\rho^{2}}\right) T_{m}+\frac{\partial^{2} T_{m}}{\partial z^{2}}+\frac{S_{m}^{\mathrm{CW}}(\rho, z)}{k(\rho)}=0 \\
-\left.k(\rho) \frac{\partial T_{m}}{\partial \rho}\right|_{\rho=R_{o}}=\left.h T_{m}\right|_{\rho=R_{o}} ; \quad-\left.k(\rho) \frac{\partial T_{m}}{\partial \rho}\right|_{\rho=R_{i}}=0 \\
\left.\frac{\partial T_{m}}{\partial z}\right|_{z=0}=\left.\frac{\partial T_{m}}{\partial z}\right|_{z=L}=0
\end{array}
$$

where $k(\rho)$ is heat conductance, and a source term $S_{m}(\rho, z)$ is defined in (10), shown at the bottom of the page.

In Fig. 4, we demonstrate the leading angular invariant $m=0$ heat-source term for the $\mathrm{HE}_{11}$ mode of a large-core ( $\left.R_{i}=330 \mu \mathrm{m}\right)$ OmniGuide fiber, assuming all values of the power in that mode and a polymer/chalcogenide glass material combination.

As in all the following graphs, we normalize the temperature scale by the maximal absorption loss of a fiber material. As we pointed out before, most of the heating comes from energy dissipation in the polymer mirror layers made of highly absorbing material. This dissipation defines a very nonuniform heat source in a radial direction, with the maxima in the polymer and the minima in the glass layers.

We further expand $T_{m}(\rho, z)=\sum_{i} T_{m}^{i}(\rho) U_{m}^{i}(z)$ into a set of basis functions $T_{m}^{i}(\rho)$ which are the eigenstates of the following operator:

$$
\begin{aligned}
\left(\frac{\partial^{2}}{\partial \rho^{2}}+\frac{1}{\rho} \frac{\partial}{\partial \rho}-\frac{m^{2}}{\rho^{2}}\right) T_{m}^{i}(\rho) & =-\gamma_{i}^{2} T_{m}^{i}(\rho) \\
-\left.k(\rho) \frac{\partial T_{m}^{i}}{\partial \rho}\right|_{\rho=R_{o}} & =\left.h T_{m}^{i}(\rho)\right|_{\rho=R_{o}} \\
-\left.k(\rho) \frac{\partial T_{m}^{i}}{\partial \rho}\right|_{\rho=R_{i}} & =0 .
\end{aligned}
$$

Eigenfunctions $T_{m}^{i}(\rho)$ are easy to construct, as in each region of constant $k(\rho), T_{m}^{i}(\rho)$ can be expanded into the Bessel functions $T_{m}^{i}(\rho)=A J_{m}\left(\gamma_{i} \rho\right)+B Y_{m}\left(\gamma_{i} \rho\right)$. These regions are then matched with each other to satisfy the condition of temperature continuity across interfaces, as well as boundary conditions at the first and the last layer interfaces. $T_{m}^{i}(\rho)$ defined by (11) will then possess very convenient orthogonality properties

$$
\int_{R_{i}}^{R_{o}} T_{m}^{i}(\rho) T_{m}^{j}(\rho) \rho \mathrm{d} \rho=\delta_{i, j} .
$$

Finally, expansion coefficients $U_{m}^{i}(z)$ can be found by solving a set of decoupled linear differential equations

$$
\begin{aligned}
& \frac{\partial^{2} U_{m}^{i}(z)}{\partial z^{2}}-\gamma_{i}^{2} U_{m}^{i}(z)+\tilde{S}_{m}^{i}(z)=0 \\
& \left.\frac{\partial U_{m}^{i}(z)}{\partial z}\right|_{z=0}=\left.\frac{\partial U_{m}^{i}(z)}{\partial z}\right|_{z=L}=0
\end{aligned}
$$

where

$$
\tilde{S}_{m}^{i}(z)=\int_{R_{i}}^{R_{o}} \frac{S_{m}^{\mathrm{CW}}(\rho, z)}{k(\rho)} T_{m}^{i}(\rho) \rho \mathrm{d} \rho .
$$

The solution of (13) is known in a closed form

$$
\begin{aligned}
U_{m}^{i}(z)=A^{i} \exp \left(\gamma_{i} z\right) & +B^{i} \exp \left(-\gamma_{i} z\right) \\
& +\frac{1}{2 \gamma_{i}} \int_{0}^{L} \exp \left(-\gamma_{i}\left|z-z^{\prime}\right|\right) \tilde{S}_{m}^{i}\left(z^{\prime}\right) \mathrm{d} z^{\prime}
\end{aligned}
$$

$$
\begin{array}{r}
S_{m}^{\mathrm{CW}}(\rho, z)=\frac{\operatorname{Im}(\varepsilon(\rho)) \omega}{4 c} \cdot \sum_{i, j}\left(\sum_{\left|m^{\prime}-m^{\prime \prime}\right|=m} C_{i, m^{\prime}}^{* \pm}(z) C_{j, m^{\prime \prime}}^{ \pm}(z) \chi^{i, j, m^{\prime}-m^{\prime \prime}} \exp \left(\left[-\operatorname{Im}\left(\beta_{j, m^{\prime \prime}}+\beta_{i, m^{\prime}}\right)+i \operatorname{Re}\left(\beta_{j, m^{\prime \prime}}-\beta_{i, m^{\prime}}\right)\right] z\right)\right. \\
\left.+\sum_{\left|m^{\prime}+m^{\prime \prime}\right|=m} C_{i, m^{\prime}}^{* \pm}(z) C_{j, m^{\prime \prime}}^{ \pm}(z) \chi^{i, j, m^{\prime}+m^{\prime \prime}} \exp \left(\left[-\operatorname{Im}\left(\beta_{j, m^{\prime \prime}}+\beta_{i, m^{\prime}}\right)+i \operatorname{Re}\left(\beta_{j, m^{\prime \prime}}-\beta_{i, m^{\prime}}\right)\right] z\right)\right) \quad(10)
\end{array}
$$




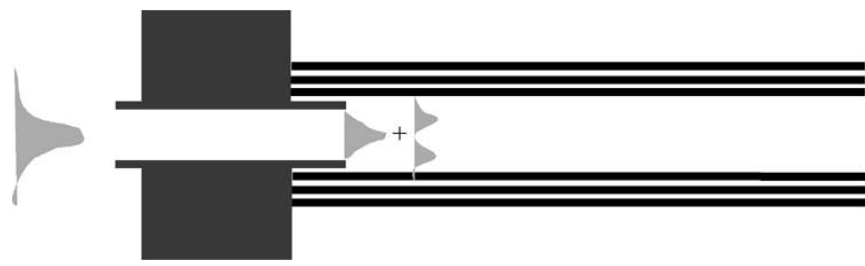

Fig. 5. High-power coupling into a fiber via a tube coupler. The metal coupler tube is inserted into a block of metal to shield an input fiber facet from direct illumination by laser beam. The coupler tube is a thin metallic tube of inner radius, close to that of a fiber core radius.

where coefficients $A^{i}$ and $B^{i}$ are chosen to satisfy the boundary conditions

$$
\left.\frac{\partial U_{m}^{i}(z)}{\partial z}\right|_{z=0}=\left.\frac{\partial U_{m}^{i}(z)}{\partial z}\right|_{z=L}=0
$$

Finally, temperature distribution across the fiber is

$$
T(\rho, \theta, z)=\sum_{i, m} T_{m}^{i}(\rho) U_{m}^{i}(z) \cos (m \theta) .
$$

Thus, the introduced method is effectively a multipole expansion method with a source for the heat-transfer equation.

\section{Heating Due to an Imperfect Coupler}

In the following, we analyze the heating of a fiber when laser light is coupled through an imperfect coupler that excites higher order modes. Fiber is designed for an optimal performance at $10.6 \mu \mathrm{m}$, having a core radius of $R_{i}=330 \mu \mathrm{m}$.

A simple coupler (Fig. 5) can be a short metallic tube inserted into a metallic block shielding an input fiber facet. Depending upon coupling conditions, such as the diameter of an incoming Gaussian beam, higher order modes in the fiber can be exited. To demonstrate the effect of modal interference on local fiber heating, we took a particular realization of a coupler where nonoptimal size of an incoming Gaussian laser beam resulted in total power being distributed $80 \%$ in $\mathrm{HE}_{11}$ mode, $10 \%$ in $\mathrm{HE}_{12}$ mode, and the rest in other high-order modes. Theoretical losses of $\mathrm{HE}_{11}$ and $\mathrm{HE}_{12}$ modes were computed to be 0.2 and $1 \mathrm{~dB} / \mathrm{m}$, respectively. Although fractional power in the $\mathrm{HE}_{11}$ mode is eight times larger than that of the $\mathrm{HE}_{12}$ mode, losses of the $\mathrm{HE}_{11}$ mode are five times smaller than the losses of the $\mathrm{HE}_{12}$ mode, leading to heat sources from the two modes that are comparable in magnitude. Because of the modal field interference, local loss can get larger or smaller than the weighted average of the two losses, leading to an appearance of "hot" spots along the fiber length. To demonstrate this effect, we solve the heat-transfer equations exactly with the modal excitation pattern due to an imperfect coupler described above and assuming the passive air cooling of a fiber. The result is presented in Fig. 6.

We plot temperature rise along a 3-m long fiber per $1 \mathrm{~W}$ of incoming light normalized by the maximal bulk-absorption material loss in a fiber. Note that the $\mathrm{HE}_{11}$ mode alone contributes to an almost-uniform temperature rise across the length of a fiber (dotted curve in Fig. 6). Inclusion of other excited modes can contribute significantly to the heating despite their small fractional power. Temperature distribution along the fiber

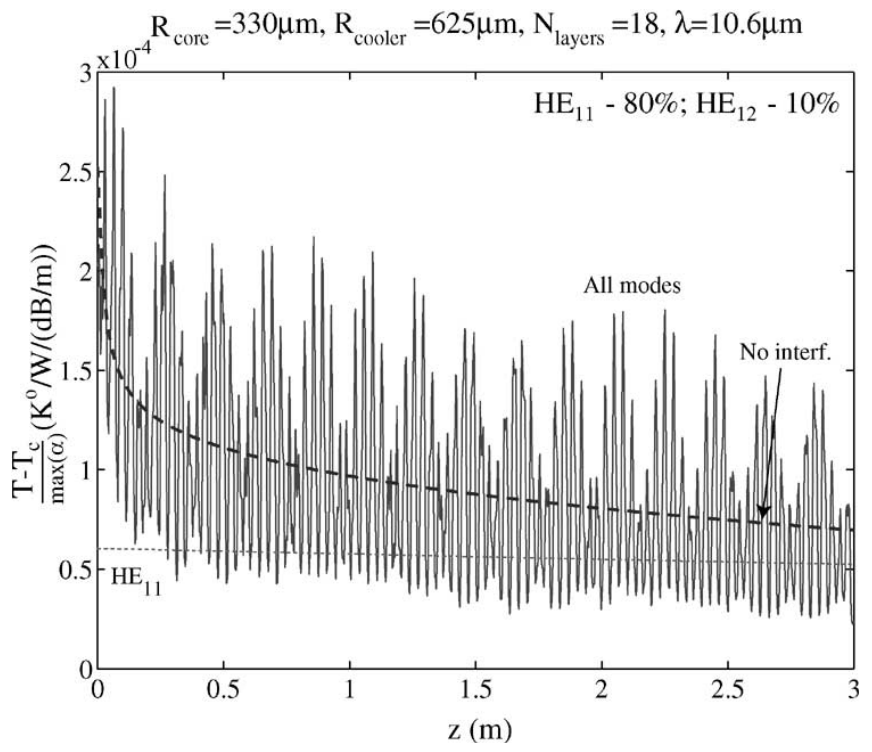

Fig. 6. Temperature rise per $1 \mathrm{~W}$ of input power along a 3-m fiber normalized by the maximum material-absorption loss of a fiber material. The dotted curve shows a temperature rise when only the $\mathrm{HE}_{11}$ mode is excited. The solid curve shows a temperature rise when several modes are excited with $80 \%$ in the $\mathrm{HE}_{11}$ mode, $10 \%$ in the $\mathrm{HE}_{12}$ mode, and with the rest of the power in higher order modes. The strongly oscillatory nature of the temperature distribution is due to interference between excited fiber modes. The thick dashed curve corresponds to the temperature distribution when the modal interference is not taken into account.

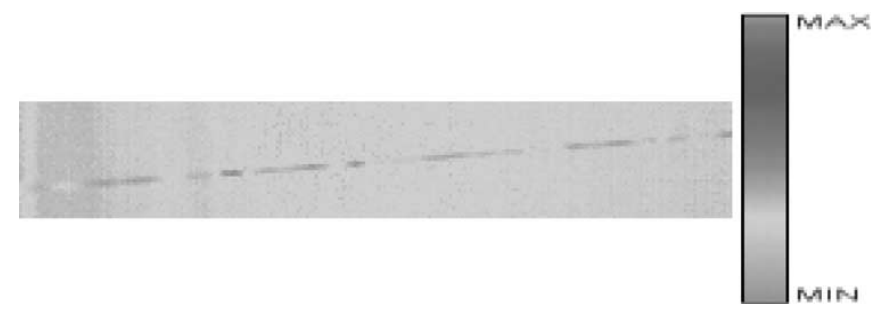

Fig. 7. IR image of a $20 \mathrm{~cm}$ fiber piece near the coupling facet. Temperature oscillations ("hot" spots) are observed every $2-5 \mathrm{~cm}$ along the fiber length.

length is very nonuniform (solid curve), with hot spots appearing every $3 \mathrm{~cm}$, corresponding approximately to a beat length between $\mathrm{HE}_{11}$ and $\mathrm{HE}_{12}$ modes. If interference effects between the modes are not taken into account, the heating profile [dashed thick curve calculated using (1)] only describes an average heat rise along the fiber length, completely missing the phenomenon of "hot" spots, which can sometimes be a dominant failure mechanism in high-power-guiding fibers. If special care is not taken when launching the light into the fiber, local "hot" spots along the fiber length can be easily detected by an $I R$ camera.

In Fig. 7, an $I R$ mage of a 20-cm-long OmniGuide fiber piece near the tube coupler is presented. "Hot" spots are clearly visible every $2-5 \mathrm{~cm}$ along the fiber length. Actually, the phenomenon of "hot" spots and their distribution along the fiber length was first predicted numerically, and then observed experimentally, in real fiber links. In fact, statistics of distribution of "hot" spots along several-meter fiber length can be used to deduce the excitation pattern of core modes. This excitation pattern can then be compared to the one deduced independently [10] from the field-intensity distributions in the near and far field regions at the fiber output. Consistent mode mixing from the 
two methods is typically observed in the absence of structural defects near the fiber output end. Another way of comparing numerical and theoretical prediction is at the fiber input end. At the coupler input facet, we use mode-matching software to predict the excitation pattern from a Gaussian laser beam into a tube coupler. This numerical excitation pattern can then be used to calculate the numerical distribution of "hot" spots along the fiber length close to the coupler region. A good agreement with experimental observations is typically found in the absence of structural defects in the coupling region.

\section{Heating Due to Bends}

When bends are present, mode conversion is induced. Modal coefficients along the length of a bend can be computed by coupled-mode theory [11]. If the bend shape is not optimally chosen, additional fiber heating can be substantial. Mode conversion in bends is stronger as the fiber core radius becomes larger, and is inversely proportional to the bend radius. As higher order modes have larger attenuations, their presence can lead to a substantial increase in the fiber heating along the bend. In Fig. 8, we demonstrate temperature rise along the $90^{\circ}$ fiber bend of a $20-\mathrm{cm}$ bend radius, assuming all of the incoming power in the $\mathrm{HE}_{11}$ mode at the start of a bend. At the beginning of the bend, before substantial mode conversion occurs, heating is dominated by the $\mathrm{HE}_{11}$ modal loss. Further into the bend, modal conversion is substantial, leading to an appreciable temperature rise. Note the appearance of "hot" spots along the bend length because of the modal interference. When compared to the temperature rise in a straight fiber, heating in an unoptimized bend can be several times larger, thus, ultimately limiting the power capacity of a high-power fiber.

\section{Fiber Heating With a Pulsed Laser Source}

We now analyze HBF power handling of pulsed radiation.

We assume $P_{\text {peak }}[\mathrm{W}]$ peak power transmitted through the waveguide with $\tau$ [s] pulse duration and $\nu[1 / \mathrm{s}]$ repetition rate. We denote the average transmitted power as $P_{\mathrm{av}}=P_{\text {peak }} \tau / \nu$. Typically, the pulse duration is much smaller than the characteristic temperature-equilibration time across the fiber cross section $\tau_{\text {eq }}=C_{v} R_{i}^{2} / k \sim 1 \mathrm{~s}$. In a regime of short pulses, temperature distribution across the fiber cross section will be modified from that of the equilibrium distribution corresponding to the average transmitted power $P_{\mathrm{av}}$. In general, power handling of pulsed radiation is more challenging than the power handling of $\mathrm{CW}$ radiation of the same average intensity. Physically, this can be attributed to excitation by the time-dependent source of localized "heat waves" (Fig. 9).

\section{A. Simple Model}

As before, we assume laser-beam coupling to the fiber modes counted by index $m$ with fractional average powers $P_{\mathrm{av}}^{m}$ in each of them. Disregarding the interference effects between the modes, the most heated region of the fiber will again be at the coupling facet of a fiber near the inner core radius. Peak temperature value $T_{i}$ in this region over time can be found by solving a simplified time-dependent heat-transfer equation with boundary conditions of zero flux across the fiber side facets

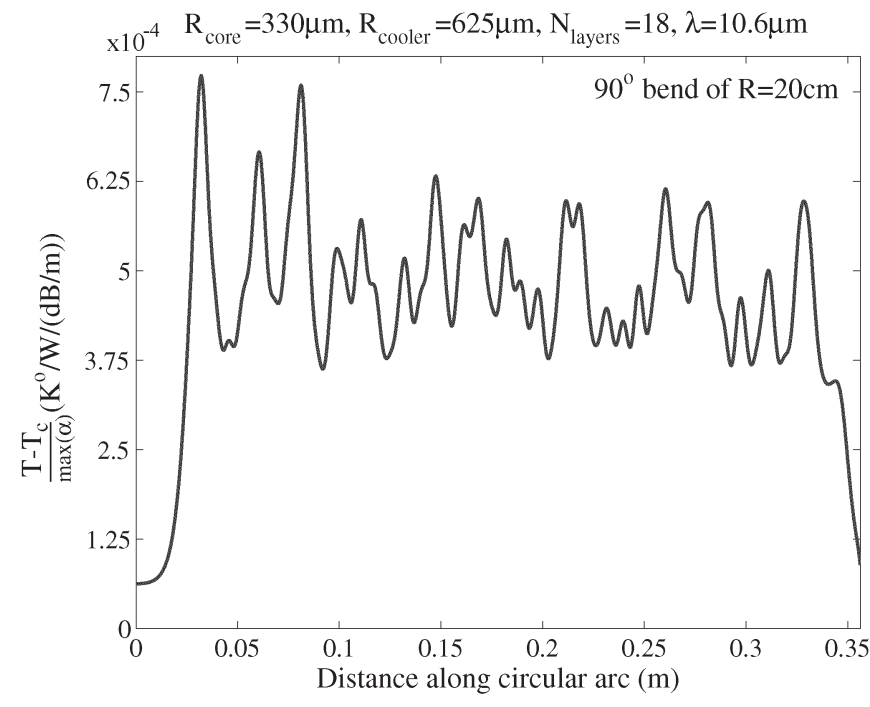

Fig. 8. Heating along a $90^{\circ}$ fiber bend of $20-\mathrm{cm}$ bend radius, assuming all of the incoming power is in the $\mathrm{HE}_{11}$ mode. At the beginning of the bend, before substantial mode conversion, heating is dominated by the $\mathrm{HE}_{11}$ modal loss. After $2 \mathrm{~cm}$ into the bend, modal conversion is substantial, leading to a significant temperature rise.

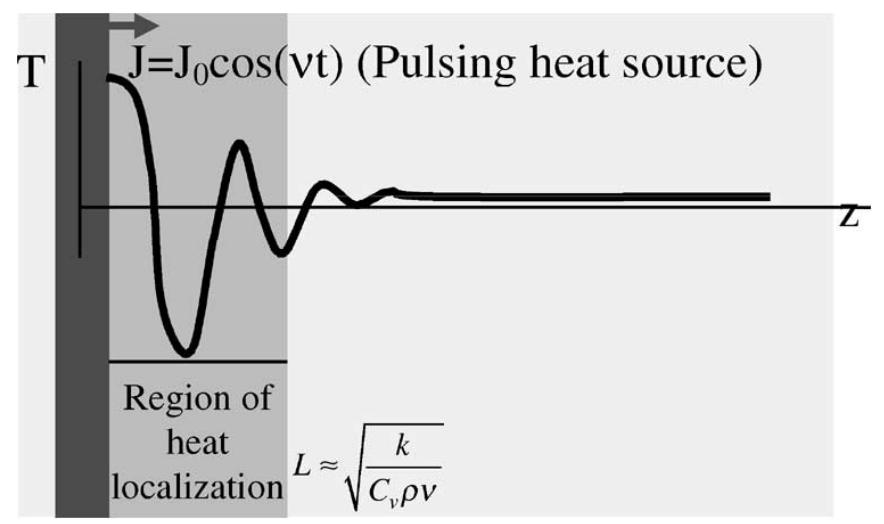

Fig. 9. Phenomenon of a "heat wave." Heat dissipation from a periodic in time heat source deteriorates as the temporal frequency of a source gets larger, leading to increased local heating. Heat from a time-dependent source localizes inside a region of size $L$, which is inversely proportional to the square root of a source frequency. The larger is the frequency (pulse repetition rate) of a heat source, the more localized is the "heat wave," leading to a deterioration of heat exchange through the outer boundary of the fiber to the coolant.

and assuming that all the heat flux was coming into the fiber from a very narrow heat source at the fiber-core boundary (we later refer to it as a delta-function heat source). The maximum temperature is then

$$
\Delta T_{i} \approx \Delta T^{\mathrm{CW}}+\left(\sum_{R_{i} \alpha_{m} \ll 1} \alpha_{m} P_{\mathrm{av}}^{m}\right)\left(\frac{1}{\nu \sqrt{\tau \tau_{\mathrm{eq}}}} \frac{0.84}{2 \pi k_{\mathrm{oclad}}}\right)
$$

where $\mathrm{CW}$ contribution $\Delta T^{\mathrm{CW}}$ is computed with (1), and $P_{\mathrm{av}}^{m}$ is fractional average powers, assuming that high-loss overcladding modes $R_{i} \alpha_{m} \gg 1$ are suppressed. For a given fiber design, the temperature rise of a pulsed source as a function of an average power, pulse duration, and pulse repetition rate can then be presented by a universal line in coordinates $\left(1 /\left(\nu \sqrt{\tau \tau_{\text {eq }}}\right), \Delta T_{i} / P_{\mathrm{av}}\right)$. In these coordinates, the temperature 
rise is given by a family of straight lines, each corresponding to a different cooling mechanism. CW power capacity is calculated by letting $1 / \nu \sqrt{\tau \tau_{\text {eq }}}=0$. Note that additional contribution to heating due to pulsed heat sources cannot be mitigated by improved cooling at the outer jacket.

\section{B. Complete Formulation}

As in the analysis of $\mathrm{CW}$ sources, we assume laser-beam coupling to a set of fiber modes counted by index $i$, angularmomentum index $m$, with excitation coefficients $C_{i, m}(z)$, in general, varying along the length of a fiber. Equilibrium temperature in this region can be found by solving a time-dependent heat-transfer equation with a boundary condition of zero flux across the fiber side facets and zero flux into the core. Heat source is computed exactly from the excited modal pattern. In the following analysis, we again assume linearly polarized modes. The vector fields of "+" and "-" polarizations of a mode with index $i$ and angular momentum $m$ are

$$
\begin{aligned}
F_{ \pm}^{i, m}(\rho, \theta, z)=W\left(\frac{z}{v_{i, m}}-t\right) & F_{ \pm}^{i, m}(\rho, \theta) C_{i, m}^{ \pm}(z) \\
& \times \exp \left(i \beta_{i, m} z-i \omega t\right)
\end{aligned}
$$

where $F_{ \pm}^{i, m}(\rho, \theta)$ are the same as in the $\mathrm{CW}$ case. We assume that the pulse is narrow enough in frequency that the fields can be computed only at a single frequency. The shape of the pulse comes through a multiplicative pulse-shape term $W\left(z / v_{i, m}-t\right)$, where $v_{i, m}$ is the group velocity of a mode $(i, m)$. Pulses are typically characterized by their repetition rate $\nu$ and duration $\tau<1 / \nu$. If pulse duration is not too short $\tau>L / v_{i, m}$, then modal intensity is approximately uniform across fiber length $L$, and additional simplification can be made by dropping the $z$ dependence of pulse shape $W(t)$. We further define $P(t)=|W(t)|^{2}$. In the case of straight fiber-expansion coefficients $C_{i, m}^{ \pm}(z)=C_{i, m}^{ \pm}$are independent of $z$ and reflect initial excitation weights, while in the case of a perturbed fiber, they are computed using coupled-mode theory. Total power flux along the fiber direction is then computed as

$$
P_{z}^{\mathrm{Pulsed}}(z, t)=P(t) P_{z}^{\mathrm{CW}}(z) .
$$

Incoming flux is normalized to reflect average power per pulse

$$
\nu \int_{0}^{\frac{1}{\nu}} P_{z}(0, t) \mathrm{d} t=P_{\mathrm{av}}[\mathrm{W}] .
$$

Heat flux from material absorption of electric field is calculated by using the imaginary part of the material dielectric constant, as in the $\mathrm{CW}$ case

$$
S^{\text {Pulsed }}(\rho, \theta, z, t)=P(t) S^{C W}(\rho, \theta, z) .
$$

We define $T(\rho, \theta, z, t)$ to be the temperature distribution across the fiber profile. Angular dependence of a $\mathrm{CW}$ heat source suggests that temperature distribution can be represented in the following form:

$$
T(\rho, \theta, z, t)=T_{c}+\sum_{m} T_{m}(\rho, z, t) \cos (m \theta)
$$

where $T_{c}$ is the coolant temperature. For each of the angular components of the temperature distribution, heat-transfer equations written in cylindrical coordinates are

$$
\begin{aligned}
& \left(\frac{\partial^{2}}{\partial \rho^{2}}+\frac{1}{\rho} \frac{\partial}{\partial \rho}-\frac{m^{2}}{\rho^{2}}\right) T_{m}+\frac{\partial^{2} T_{m}}{\partial z^{2}} \\
& \quad+P(t) \frac{S_{m}^{\mathrm{CW}}(\rho, z)}{k(\rho)}=\frac{C(\rho)}{k(\rho)} \frac{\partial T_{m}}{\partial t} \\
& -\left.k(\rho) \frac{\partial T_{m}}{\partial \rho}\right|_{\rho=R_{o}}=\left.h T_{m}\right|_{\rho=R_{o}} ; \quad-\left.k(\rho) \frac{\partial T_{m}}{\partial \rho}\right|_{\rho=R_{i}}=0 \\
& \left.\frac{\partial T_{m}}{\partial z}\right|_{z=0}=\left.\frac{\partial T_{m}}{\partial z}\right|_{z=L}=0
\end{aligned}
$$

where $k(\rho)$ is heat conductance, and $C(\rho)$ is heat capacitance per unit volume. We further make an expansion

$$
T_{m}(\rho, z, t)=\sum_{i} T_{m}^{i}(\rho) U_{m}^{i}(z, t)
$$

into the same set of basis functions $T_{m}^{i}(\rho)$, as in a CW case. Using the orthogonality property (12) of these functions, $U_{m}^{i}(z, t)$ can then be found by solving a set of decoupled differential equations

$$
\begin{aligned}
& \frac{\partial^{2} U_{m}^{i}(z, t)}{\partial z^{2}}-\gamma_{i}^{2} U_{m}^{i}(z, t)+P(t) \tilde{S}_{m}^{i}(z)=\frac{C}{k} \frac{\partial U_{m}^{i}(z, t)}{\partial t} \\
& \left.\frac{\partial U_{m}^{i}(z, t)}{\partial z}\right|_{z=0}=\left.\frac{\partial U_{m}^{i}(z, t)}{\partial z}\right|_{z=L}=0
\end{aligned}
$$

where $\tilde{S}_{m}^{i}(z)$ is the same as in a CW case, and the $C(\rho) / k(\rho)$ ratio is assumed to be constant across the fiber profile (this assumption can be lifted, leading to a system of coupled differential equations in index $i$ ).

We now make another expansion

$$
U_{m}^{i}(z, t)=\sum_{n=0}^{\infty} \cos \left(\alpha_{n} z\right) D_{m}^{i, n}(t)
$$

where $\alpha_{n}=\pi n / L$ to satisfy boundary conditions at the fiber ends. Equations (26) are then simplified

$$
\frac{\partial D_{m}^{i, n}(t)}{\partial t}+\frac{k}{C}\left(\gamma_{i}^{2}+\alpha_{n}^{2}\right) D_{m}^{i, n}(t)=\frac{k}{C} P(t) \tilde{S}_{m}^{i, n}
$$

where we introduce

$$
\tilde{S}_{m}^{i, n}=\frac{\xi_{n}}{L} \int_{0}^{L} \cos \left(\alpha_{n} z\right) \tilde{S}_{m}^{i}(z) \mathrm{d} z
$$

and $\xi_{0}=1 ; \xi_{m \neq 0}=2$. We are interested in the temporally periodic solution of (28) corresponding to a stationary solution of the heat transfer-problem. Such a solution is known in a closed form as

$$
D_{m}^{i, n}(t)=\frac{k}{C} \tilde{S}_{m}^{i, n} \int_{-\infty}^{t} \exp \left(-\frac{k\left(\gamma_{i}^{2}+\alpha_{n}^{2}\right)}{C}\left(t-t^{\prime}\right)\right) P\left(t^{\prime}\right) \mathrm{d} t^{\prime} .
$$




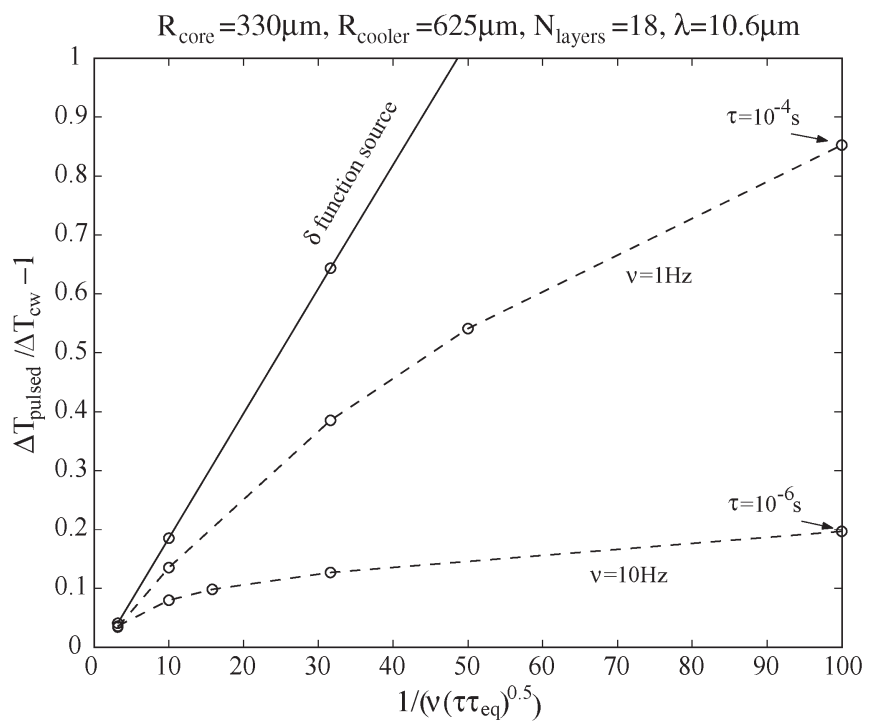

Fig. 10. Temperature-rise parameter curves as a function of a laser-pulse parameter. Different dashed curves are calculated for an exact heat source and correspond to different pulse-repetition rates. The solid curve is calculated for a simplistic $\delta$ function like heat source, which provides an upper bound on the heating increase due to pulsed sources.

In the case of a square pulse, $P(t)$ is a periodic function with a period $1 / \nu$ defined in the interval $(0,1 / \nu)$ as

$$
P(t)=P_{\text {av }} \frac{1}{\tau \nu}\left[\begin{array}{l}
1 ; 0<t<\tau \\
0 ; \tau<t<\frac{1}{\nu}
\end{array} .\right.
$$

Finally, temperature distribution across the fiber is

$$
T(\rho, \theta, z, t)=\sum_{i, n, m} T_{m}^{i}(\rho) \cos \left(\alpha_{n} z\right) \cos (m \theta) D_{m}^{i, n}(t) .
$$

Detailed calculation of pulsed sources of the form (31) shows that temperature rise is actually smaller when evaluated realistically, rather then a $\delta$ function like heat source [expression (18)]. As the repetition rate of the pulse increases, for the same value of a parameter $1 /\left(\nu \sqrt{\tau \tau_{e q}}\right)$ temperature distribution tends to get closer to the $\mathrm{CW}$ distribution. In Fig. 9, we present an increase in the maximal temperature rise due to a pulsed source compared to a temperature increase in a $\mathrm{CW}$ case $\Delta T / \Delta T_{\mathrm{CW}}-1$ as a function of a pulse parameter $1 /\left(\nu \sqrt{\tau \tau_{e q}}\right)$ for a single excited mode. Simplified theory with a $\delta$ function like heat source predicts a straight solid line in Fig. 10. Detailed calculations show that this line gives an upper bound on the heating due to pulsed sources. In reality, because of the finite extent of a heat source, temperature-rise curves tend to follow a $\mathrm{CW}$ temperature rise as the pulse repetition rate increases. In Fig. 10, several temperature-rise curves are presented. They are labeled and calculated for particular values of pulse repetition rates $\nu$ and varying pulse duration $\tau$.

\section{Degradation of BeAm QuAlity $-M^{2}$}

For the completeness of the discussion, we mention in passing additional restrictions on high-power transmission-system design due to the degradation of spatial beam quality. Although not directly related to failure mechanisms due to heating, degradation of a beam quality imposes additional requirements on the uniformity of a transmission link, minimal bend radii allowed, etc. As the concept of beam quality plays major role in industrial applications, it deserves a special paper of its own. Here, we will only introduce major concepts related to the issue of beam quality in HBF links.

Degradation of the spatial content of propagating radiation through an imperfect fiber is frequently characterized by the beam-quality parameter $M^{2}$, which measures the divergence of a given beam of radiation relative to the divergence of a minimum-divergence Gaussian beam in the form of

$$
D_{M^{2}}^{2}(z)=D^{2}(0)\left[1+\left(\frac{4 M^{2} \lambda z}{\pi D^{2}(0)}\right)^{2}\right]^{\frac{1}{2}} .
$$

This measure ultimately reflects the smallest spot size to which an outgoing beam can be focused. To estimate $M^{2}$, one fits $D_{M^{2}}(z)$ to a numerically simulated beam divergence defined through the simulated field intensity $I(r, z)$ as

$$
D(z)=2 \sqrt{2}\left[\frac{\oint r^{2} I(r, z) \mathrm{d} A}{\oint I(r, z) \mathrm{d} A}\right]^{\frac{1}{2}}
$$

Beam diameter $D(z)$ is simulated by numerically propagating the field from an output of a fiber to the distance $L$ (usually several centimeters) until a reliable determination of $M^{2}$ is achieved.

The main mechanism for beam degradation is intermodal scattering into the higher order modes by micro and macro imperfections along the propagation length. Micro imperfections include fiber core roughness, acircularity of a core, microbends, etc., while macro imperfections typically include various fiber curvature changes. Modal scattering leads to an increase in the output beam diameter, an increase in the angle of beam divergence, and "speckling" of the radiation profile. In the case when most of the power is in a single mode, $M^{2}$ can be approximated as an $M^{2}$ of that mode plus $M^{2}$ numbers of the other modes weighted by their fractional power in a beam. Fractional powers in other modes can be calculated given a particular profile of a fiber along its length by coupled-mode theory. $M^{2}$ for a general beam cannot be readily evaluated from the $M^{2}$ of the constituent modes as there are interference terms between the modes that are not taken into account by the simple addition of $M^{2}$ numbers. As HBFs are multimoded, individual modes can have significantly different qualities when focused outside the fiber. For reference, we present results of the simulation of $M^{2}$ for several major modes in the $\mathrm{HBF}$ of chalco/polymer material combination with $\mathrm{ID}=660 \mu \mathrm{m}$, $\mathrm{OD}=1250 \mu \mathrm{m}$, and 20 bilayers.

\begin{tabular}{|c|c|c|c|c|c|}
\hline HE11 & TE01 & HE21 & HE12 & EH11 & EH21 \\
\hline 1.10 & 2.17 & 2.17 & 3.02 & 3.22 & 4.26 \\
\hline
\end{tabular}

\section{CONCLUSION}

In this paper, we have developed an efficient yet exact semianalytical numerical technique to investigate heating from continuous-wave $(\mathrm{CW})$ and pulsed-radiation propagation, coupling, and bending in an emerging new type of high-powerradiation guides - hollow Bragg fibers (HBFs). The main factor 
limiting the power capacity of high-power HBF links is found to be additional heating due to fundamental mode scattering into the lossy higher order modes. Such scattering is typically caused by imperfect coupling at the coupling end, micro imperfections, and macro bending along the fiber-propagation length. When several modes are excited in the fiber, the phenomenon of "hot" spots is observed due to modal interference, when even a small partial content of a very lossy mode can lead to a discernable beating pattern in temperature distribution with pronounced temperature maxima. Finally, for the propagation of pulsed radiation, additional heating is observed due to heat localization near the pulsed heat source. This causes additional heating in the first few layers of a reflector, which is difficult to mediate by improving the outside cooling conditions.

\section{REFERENCES}

[1] P. Yeh, A. Yariv, and E. Marom, "Theory of Bragg fiber," J. Opt. Soc. Amer., vol. 68, no. 9, pp. 1196-1201, Sep. 1978.

[2] J. A. Harrington, "A review of IR transmitting, hollow waveguides," Fiber Integr. Opt., vol. 19, no. 3, pp. 211-227, Jul. 2000.

[3] Y. Matsuura and M. Miyagi, "Hollow fibers for high-power lasers," in Proc. SPIE, San Jose, CA, 1999, vol. 3609, p. 251.

[4] B. Temelkuran, S. D. Hart, G. Benoit, J. D. Joannopoulos, and Y. Fink, "Wavelength-scalable hollow optical fibres with large photonic bandgaps for $\mathrm{CO}_{2}$ laser transmission," Nature, vol. 420, no. 6916, p. 650, Dec. 2002.

[5] H. Sabert, "Hollow-core fibers allow light to travel by air," Laser Focus World, vol. 40, no. 5, pp. 161-164, May 2004.

[6] K. Lyytikainen, J. Zagari, G. Barton, and J. Canning, "Heat transfer within a microstructured polymer optical fibre perform," Model. Simul. Mater. Sci. Eng., vol. 12, no. 3, pp. 255-265, May 2004.

[7] R. K. Nubling and J. A. Harrington, "Thermal and modal properties of hollow-glass waveguides," in Proc. SPIE, San Jose, CA, 1997, vol. 2977, p. 51.

[8] D. Su, S. Somkuarnpanit, D. R. Hall, and J. D. C. Jones, "Thermal effects in a hollow waveguide beam launch for $\mathrm{CO}_{2}$ laser power delivery," Appl. Opt., vol. 35, no. 24, pp. 4787-4789, Aug. 1996.

[9] S. Yi-Wei, P. Zhi-Yun, Y. Matsuura, Y. Konno, Y. Aizawa, and M. Miyagi, "Properties of hollow bent output tips in $\mathrm{CO}_{2}$ laser light delivery system," Opt. Laser Technol., vol. 33, no. 4, pp. 225-230, 2001.
[10] M. Skorobogatiy, C. Anastassiou, S. G. Johnson, O. Weisberg, T. D. Engeness, S. A. Jacobs, R. U. Ahmad, and Y. Fink, "Quantitative characterization of higher-order mode converters in weakly multimoded fibers," Opt. Express, vol. 11, no. 22, pp. 2838-2847, Nov. 2003.

[11] S. G. Johnson, M. Ibanescu, M. Skorobogatiy, O. Weisberg, T. D. Engeness, M. Soljacic, S. A. Jacobs, J. D. Joannopoulos, and Y. Fink, "Low-loss asymptotically single-mode propagation in large-core OmniGuide fibers," Opt. Express, vol. 9, no. 13, p. 748, Dec. 2001.

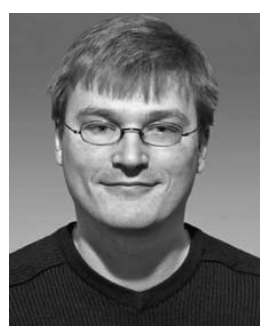

M. Skorobogatiy received the M.Sc. degree in electrical engineering and computer science in 2000 and the Ph.D. degree in physics in 2001, both from the Massachusetts Institute of Technology, Cambridge.

$\mathrm{He}$ then joined MIT start-up OmniGuide Inc., where he worked as a theory and simulations group leader developing hollow Bragg fibers for high power laser guiding applications. Since 2003, he has been a professor and a Canada Research Chair in photonic crystals with the Department of Engineering Physics, École Polytechnique de Montréal, Montréal, QC, Canada. His current interests include photonic crystal fiber fabrication and modeling as well as photonic crystals in general.

S. A. Jacobs, photograph and biography not available at the time of publication.

S. G. Johnson, photograph and biography not available at the time of publication.

C. Anastassiou, photograph and biography not available at the time of publication.

B. Temelkuran, photograph and biography not available at the time of publication. 